\title{
The Periodic Solutions to Kawahara Equation by Means of the Auxiliary Equation with a Sixth-Degree Nonlinear Term
}

\author{
Zehra Pınar and Turgut Öziş \\ Department of Mathematics, Science Faculty, Ege University, Bornova, 35100 Izmir, Turkey \\ Correspondence should be addressed to Zehra Pınar; zehra.pnar@gmail.com
}

Received 15 August 2012; Accepted 23 September 2012

Academic Editor: Anjan Biswas

Copyright (C) 2013 Z. Pınar and T. Öziş. This is an open access article distributed under the Creative Commons Attribution License, which permits unrestricted use, distribution, and reproduction in any medium, provided the original work is properly cited.

It is well known that different types of exact solutions of an auxiliary equation produce new types of exact travelling wave solutions to nonlinear equations. In this paper, by means of symbolic computation, the new solutions of original auxiliary equation of firstorder nonlinear ordinary differential equation with a sixth-degree nonlinear term are presented to obtain novel exact solutions of the Kawahara equation. By the aid of the solutions of the original auxiliary equation, some other physically important nonlinear equations can be solved to construct novel exact solutions.

\section{Introduction}

Over the past decades a number of approximate methods for finding travelling wave solutions to nonlinear evolution equations have been proposed or developed and furthermore modified. The solutions to various evolution equations have been found by one or other of these methods. References [124] and references within cite some of this accomplishment. Among these methods, one of the current methods is so called auxiliary equation method [20-24]. The technique of this method consist of the solutions of the nonlinear evolution equations such that the target solutions of the nonlinear evolution equations can be expressed as a polynomial in a linearly independent elementary function which satisfies a particular ordinary differential equation which is named as auxiliary equation in general. Recently, to determine the solutions of nonlinear evolution equations, many exact solutions of various auxiliary equations have been utilized [20-32].

A concise observation shows that novel exact solutions of the auxiliary equations may fabricate original exact travelling wave solutions of nonlinear equations. Therefore, it is very important to find novel exact solutions of the auxiliary equations.

In this paper, we will examine the consequences of the choice of the auxiliary equation for determining the solutions of the nonlinear evolution equation in consideration and to seek more types of new exact solutions of nonlinear differential equations which satisfy a first-order nonlinear ordinary differential equation with a sixth-degree nonlinear term.

\section{Auxiliary Equation Technique and Some Remarks}

We assume that the given nonlinear partial differential equation for $u(x, t)$ to be in the form

$$
P\left(u, u_{x}, u_{t}, u_{x x}, u_{x t}, u_{t t}, \ldots\right)=0,
$$

where $P$ is a polynomial in its arguments, which include nonlinear terms and the highest-order derivatives.

Then using the transformation $u(x, t)=u(\xi), \xi=\mu(x-$ $c t)$ one can reduce (1) to the ordinary differential equation

$$
Q\left(u, u_{\xi}, u_{\xi \xi}, u_{\xi \xi \xi}, \ldots\right)=0 .
$$

Now, we seek the exact travelling wave solutions of (2) by means of auxiliary equation method.

As it is well known, most exact travelling wave solutions [4-24] of nonlinear evolution equations were obtained on the assumption that the exact solution can be expressed as a finite expansion of linearly independent elementary functions. One common way of obtaining linearly independent elementary functions is to assume them as a solution of appropriate algebraic or differential equations which are exactly solvable. 
Thus, it is natural to ask the following question: What type of finite expansion or what kind of function can be used to construct new travelling wave solutions?

Hence, for the solution of (2), let us assume that the exact solution can be expressed as simple expansion in the form of

$$
u(\xi)=\sum_{i=0}^{N} a_{i} z^{i}(\xi)
$$

where $a_{i},(i=0,1, \ldots, N)$ are constants which will be determined later and function $z(\xi)$ is an appropriate function that yields new travelling wave solutions and is defined by the solution of the auxiliary equation considered.

Now, let us remember the process for finding the unknown coefficients $a_{i},(i=0,1, \ldots, N)$, where $u(\xi)=$ $\sum_{i=0}^{N} a_{i} z^{i}(\xi)$. Substituting the auxiliary equation into the given nonlinear equation and equating each coefficient of a power of $z(\xi)$ to zero yield an algebraic system. Hence, all coefficients $a_{i}$ can be determined by solving the algebraic system and the parameter $N$ is a positive integer and can be determined by balancing the highest order derivative terms and the highest power nonlinear terms in (3) in general.

In most auxiliary equation methods, the function $z(\xi)$ is defined as the solution of an auxiliary ordinary differential equation. Even if it is possible to construct more complex types of exact solutions for many auxiliary ordinary differential equations, one also encounters some difficulties. Hence, the following vital question arise: How do we find a variety of exact solutions of the auxiliary equations? Unfortunately, the answer of this question is not unique and various authors utilize different auxiliary ordinary differential equations which are exactly solvable.

For example, Nickel [25] has utilized the nonlinear differential equation

$$
\left(\frac{d z}{d \xi}\right)^{2}=\alpha z^{4}+4 \beta z^{3}+6 \gamma z^{2}+4 \delta z+\varepsilon \equiv R(z)
$$

as an auxiliary ordinary differential equation which transforms (2) into polynomial equation $P(z)=0, z^{\prime} Q(z)=0$, and $P(z)+z^{\prime} Q(z)=0$, respectively. Vanishing coefficients in the polynomials $P(z)=0, z^{\prime} Q(z)=0$ and $P(z)+z^{\prime} Q(z)=0$, respectively, lead to equations which partially determine the coefficients $\alpha, \beta, \gamma, \delta$, and $\varepsilon$ in (4). We think that Nickel preferred (4) due to it is analytically solvability. Because, Whittaker and Watson [26] showed that the solution $z(\xi)$ of (4) can be expressed in terms of Weierstrass' elliptic function.

At the same time, Fan [27] developed a new direct algebraic method to seek additional types of new exact solutions of evolutionary nonlinear differential equations that can be expressed as polynomials in several elementary or special functions which satisfy a first-order nonlinear ordinary differential equation with a fourth-degree nonlinear term

$$
\left(\frac{d z}{d \xi}\right)^{2}=a_{0}+a_{1} z(\xi)+a_{2} z^{2}(\xi)+a_{3} z^{3}(\xi)+a_{4} z^{4}(\xi)
$$

where $a_{i},(i=0,1,3,4)$ are constants. The method has been applied to find many exact traveling wave solutions of various types of nonlinear equations [27-29]. Sirendaorejii [21] and Abdou [30] utilized (5) for the case $a_{i},(i=2,3,4)$. Perhaps, it is significant to point out that the choice of the case, that is, $a_{i}(i=2,3,4)$, permits analytical solution of the first-order nonlinear ordinary differential equation with a fourth-degree nonlinear term as Nickel's. This property of the nonlinear ordinary differential equation with a fourth-degree nonlinear term used in large in $[20,23]$, for example, for the case $a_{i}(i=$ $0,2,4)$ and in $[21,22]$ for the case $a_{i}(i=2,3,4)$ for (5) to find many exact traveling wave solutions of various types of nonlinear equations.

In this paper, we seek for the solution of (2) in terms of ansatz (3) with $z(\xi)$ satisfying the following new auxiliary equation with a sixth-degree nonlinear term, that is,

$$
\left(\frac{d z}{d \xi}\right)^{2}=a_{0}+a_{2} z^{2}(\xi)+a_{4} z^{4}(\xi)+a_{6} z^{6}(\xi)
$$

where $a_{i}(i=0,2,4,6)$ are real constants. Cases $1-8$ report the new solutions of the auxiliary equation (6) with a sixth-degree nonlinear term under 8 distinct cases which include solutions including Bessel function and Lambert function and one can use these new solutions to seek exact travelling wave solutions for nonlinear equations.

Case 1. If

$$
\left(\frac{d z}{d \xi}\right)^{2}=a_{0}+a_{6} z^{6}(\xi)
$$

then

$$
z(\xi)=\operatorname{Bessel} J\left(\frac{1}{6}, 2 z^{6}\right) z \pi+3 \xi \Gamma\left(\frac{5}{6}\right) .
$$

Case 2. If

$$
\left(\frac{d z}{d \xi}\right)^{2}=a_{4} z^{4}(\xi)+a_{6} z^{6}(\xi)
$$

then

$$
z(\xi)=\frac{\sqrt{\left(-a_{6}+a_{4}^{2} \xi^{2}-2 a_{4}^{2} \xi_{c_{1}}+a_{4}^{2} c_{1}^{2}\right) a_{4}}}{-a_{6}+a_{4}^{2} \xi^{2}-2 a_{4}^{2} \xi_{c_{1}}+a_{4}^{2} c_{1}^{2}} .
$$

Case 3. If

$$
\left(\frac{d z}{d \xi}\right)^{2}=a_{0}+a_{2} z^{2}(\xi)+a_{6} z^{6}(\xi)
$$

then 


$$
\begin{aligned}
= & \frac{\left(\left(-(3 / 8) \xi\left(\sqrt{-10 a_{6} a_{2}} a_{2} / a_{6}\right)^{3 / 2}+(3 / 8)\left(\sqrt{-10 a_{6} a_{2}} a_{2} / a_{6}\right)^{3 / 2} c_{1}+a_{0} \sqrt{a_{0}\left(-8+9 a_{2} \xi^{2}-18 a_{2} \xi c_{1}+9 a_{2} c_{1}^{2}\right) / a_{2} / 8 a_{6}}\right) a_{2}^{2}\right)^{1 / 3}}{a_{2}} \\
& +\frac{\sqrt{-10 a_{6} a_{2}} a_{2}}{\left(2 a _ { 6 } \left(-(3 / 8) \xi\left(\sqrt{-10 a_{6} a_{2}} a_{2} / a_{6}\right)^{3 / 2}+(3 / 8)\left(\sqrt{-10 a_{6} a_{2}} a_{2} / a_{6}\right)^{3 / 2} c_{1}+a_{0} \sqrt{\left.\left.a_{0}\left(-8+9 a_{2} \xi^{2}-18 a_{2} \xi c_{1}+9 a_{2} c_{1}^{2}\right) / a_{2} / 8 a_{6}\right) a_{2}^{2}\right)^{1 / 3}}\right.\right.} .
\end{aligned}
$$

Case 4. If

$$
\left(\frac{d z}{d \xi}\right)^{2}=a_{2} z^{2}(\xi)+a_{4} z^{4}(\xi)+a_{6} z^{6}(\xi)
$$

then

$$
\begin{aligned}
z(\xi)= & e^{\left(\sqrt{a_{2}}\left(-2 \xi+c_{1}\right)\right)} \sqrt{2} \\
& \times 2\left(-\frac{a_{6} e^{\left(2 \sqrt{a_{2}}\left(-2 \xi+c_{1}\right)\right)}}{a_{4} \text { Lambert } W\left(-2 a_{6} e^{\left(2 \sqrt{a_{2}}\left(-2 \xi+c_{1}\right)\right)} / 3 a_{4}\right)}\right)^{-1 / 2} .
\end{aligned}
$$

Case 5. If

$$
\left(\frac{d z}{d \xi}\right)^{2}=a_{0}+a_{4} z^{4}(\xi)+a_{6} z^{6}(\xi)
$$

then

$$
z(\xi)=\xi-\int^{z(\xi)} \frac{d a}{\sqrt{a_{0}+a_{4} a^{4}+a_{6} a^{6}}}-c_{1}=0
$$

Case 6. If

$$
\left(\frac{d z}{d \xi}\right)^{2}=a_{2} z^{2}(\xi)+a_{6} z^{6}(\xi)
$$

then

$$
\begin{aligned}
z(\xi)= & e^{\left(\sqrt{a_{2}}\left(-\xi+c_{1}\right)\right)} 2^{1 / 4} \\
& \times\left(\frac{a_{6} e^{\left(4 \sqrt{a_{2}}\left(-\xi+c_{1}\right)\right)}}{a_{2} \text { Lambert } W\left(-a_{6} e^{\left(4 \sqrt{a_{2}}\left(-\xi+c_{1}\right)\right)} / 2 a_{2}\right)}\right)^{-1 / 4} .
\end{aligned}
$$

Case 7. If

$$
\left(\frac{d z}{d \xi}\right)^{2}=a_{6} z^{6}(\xi)
$$

then

$$
z(\xi)=\frac{1}{\sqrt{-2 \xi \sqrt{a_{6}}+2 c_{1} \sqrt{a_{6}}}} .
$$

Case 8. If

$$
\left(\frac{d z}{d \xi}\right)^{2}=a_{0}+a_{2} z^{2}(\xi)+a_{4} z^{4}(\xi)+a_{6} z^{6}(\xi)
$$

then

$$
z(\xi)
$$

$$
=\left(a _ { 6 } 8 1 ^ { 2 / 3 } \left(\left(\left(-\frac{3}{8} \xi a_{0}^{3 / 2}+\frac{3}{8} a_{0}^{3 / 2} c_{1}+\frac{3 \sqrt{8} a_{0}}{8}\right.\right.\right.\right.
$$

$$
\left.\times \sqrt{\frac{a_{0}\left(-8 a_{6}+9 a_{4}^{2} \xi^{2}-18 a_{4}^{2} \xi c_{1}+9 a_{4}^{2} c_{1}^{2}\right)}{a_{4}^{2}}}\right)
$$

$$
\left.\left.\left.\times a_{4}^{4}\right)\left(a_{6}^{2}\right)^{-1}\right)^{1 / 3}\right)\left(18 a_{4}^{2}\right)^{-1}
$$

$$
+a_{0} 81^{1 / 3}\left(2 \left(\left(-\frac{3}{8} \xi a_{0}^{3 / 2}+\frac{3}{8} a_{0}^{3 / 2} c_{1}+\frac{3 \sqrt{8} a_{0}}{8}\right.\right.\right.
$$

$$
\left.\times \sqrt{\frac{a_{0}\left(-8 a_{6}+9 a_{4}^{2} \xi^{2}-18 a_{4}^{2} \xi c_{1}+9 a_{4}^{2} c_{1}^{2}\right)}{a_{4}^{2}}}\right)
$$

$$
\left.\left.\times a_{4}^{4}\right)\left(a_{6}^{2}\right)^{-1}\right)^{-1 / 3}
$$

As it is noticeable, by choosing the particular values of $a_{i}(i=0,2,4,6),(6)$ provides numerous types of special solutions. Due to limited space, we omit those results; instead we affirm the following remarks.

Hence, in ansatz (3), the parameter $N$ is a positive integer and can be determined by balancing the higher-order 
derivative term and the highest power nonlinear terms in (2). The highest degree of $\partial^{p} u / \partial \xi^{p}$ can be calculated by

$$
\begin{gathered}
\mathcal{O}\left[\frac{\partial^{p} u}{\partial \xi^{p}}\right]=N+p, \quad p=0,1,2, \ldots, \\
\mathcal{O}\left[u^{q} \frac{\partial^{p} u}{\partial \xi^{p}}\right]=q N+p, \quad q, p=0,1,2, \ldots
\end{gathered}
$$

Consequently substituting (3) and (6) into (2) and equating the coefficients of all powers of $z(\xi)$ and $z^{j}(\xi) \sqrt{a_{0}+a_{2} z^{2}(\xi)+a_{4} z^{4}(\xi)+a_{6} z^{6}(\xi)}(j=0,1,2, \ldots)$ to zero in the resulting equation, several algebraic equations will be obtained. Then solving these algebraic equations by the symbolic computation system Maple, and combining (3) and the auxiliary equation (6), we can get the exact solutions for (1).

\section{Exact Travelling Wave Solutions}

In this section, we are going to combine the auxiliary equation method with the new solutions of original auxiliary equation (6) with a six-degree nonlinear term to consider the new solutions of the generalized nonlinear Klein-Gordon equation which is used as a test problem by a number of researchers $[20,21,24]$.

The Kawahara equation is

$$
u_{t}+u u_{x}+\alpha u_{x x x}-u_{x x x x x}=0,
$$

where $\alpha$ is nonzero arbitrary constant. Equation (24), proposed first by Kawahara in 1972, occurs in the theory of shallow water waves and plays an important role in the modeling of many physical phenomena such as plasma waves and magnetoacoustic waves. Making the transformation $u(x, t)=$ $u(\xi), \xi=x-c t$ and integrating once with respect to $\xi,(24)$ becomes

$$
-c u+\frac{1}{2} u^{2}+\alpha u^{\prime \prime}-u^{(4)}=0 .
$$

From (23), we have $N=4$. Therefore, the ansatz yields

$$
u(\xi)=g_{0}+g_{1} z(\xi)+g_{2} z^{2}(\xi)+g_{3} z^{3}(\xi)+g_{4} z^{4}(\xi)
$$

and $z(\xi)$ may be determined from one of the Cases 1-8.

Now, for convenience, we give the calculation of typical two cases only for the practical purposes and the rest can be determined in a similar manner.

Example 1. We first consider the auxiliary equation with sixdegree nonlinearity, that is,

$$
\left(\frac{d z}{d \xi}\right)^{2}=a_{4} z^{4}(\xi)+a_{6} z^{6}(\xi) .
$$

in Cases 1-8. The solution of (27)

$$
z(\xi)=\frac{\sqrt{\left(-a_{6}+a_{4}^{2} \xi^{2}-2 a_{4}^{2} \xi c_{1}+a_{4}^{2} c_{1}^{2}\right) a_{4}}}{-a_{6}+a_{4}^{2} \xi^{2}-2 a_{4}^{2} \xi c_{1}+a_{4}^{2} c_{1}^{2}}
$$

is, we belive, a new solution in the literature.
Hence, substituting (26) and (27) into (25) and letting each coefficient of $z^{i}(\xi) \sqrt{a_{2} z^{2}(\xi)+a_{6} z^{6}(\xi)},(0 \leq i \leq 8)$ to be zero, we obtain

$$
\begin{gathered}
-384 g_{2} a_{6}-2688 g_{4} a_{4}=0, \\
-105 g_{1} a_{6}-1260 g_{3} a_{4}=0, \\
24 a_{6} \alpha g_{4}+\frac{1}{2} g_{4}^{3}-840 g_{4} a_{4}^{2}-480 a_{6} a_{4} g_{2}=0, \\
15 a_{6} \alpha g_{3}+g_{3} g_{4}-360 g_{3} a_{4}^{2}-120 a_{6} a_{4} g_{1}=0, \\
20 a_{4} \alpha g_{4}+\frac{1}{2} g_{3}^{2}-120 g_{2} a_{4}^{2}+8 a_{6} \alpha g_{2}+a g_{4} g_{2}=0, \\
3 a_{6} \alpha g_{1}+g_{1} g_{4}+g_{2} g_{3}-24 g_{1} a_{4}^{2}+12 \alpha a_{4} g_{3}=0, \\
g_{0} g_{4}+g_{1} g_{3}-c g_{4}+\frac{1}{2} g_{2}^{2}+6 \alpha a_{4} g_{2}=0, \\
g_{0} g_{3}+g_{1} g_{2}-c g_{3}+2 \alpha a_{4} g_{1}=0, \\
g_{0} g_{2}-c g_{2}+\frac{1}{2} g_{1}^{2}=0, \\
g_{0}-c=0 .
\end{gathered}
$$

Solving the system (29) by the aid of Maple 13, we can determine the coefficients:

$$
\begin{gathered}
g_{0}=\frac{4 c}{3}, \quad g_{2}=g_{1}=g_{3}=0, \quad \mu=\mu, \\
a_{4}=a_{4}, \quad a_{6}=a_{6}, \\
c=8064 a_{4}-72 \alpha a_{6}-\frac{3}{2} g_{4}+2520 a_{4}^{2}-60 \alpha a_{4} .
\end{gathered}
$$

Substituting the above coefficients into ansatz (26) with the solution (28) of auxiliary equation, we obtain one of the new solutions of Kawahara equation:

$$
\begin{aligned}
u(x, t):= & 10752 a_{4}-96 \alpha a_{6}-2 g_{4}+3360 a_{4}^{2} \\
& -80 a_{4} \alpha+g_{4} a_{4}^{2} \\
& \times\left(-a_{6}+a_{4}^{2}\left(x+\left(8064 a_{4}-72 \alpha a_{6}\right.\right.\right. \\
& \left.\left.-\frac{3}{2} g_{4}+2520 a_{4}^{2}-60 a_{4} \alpha\right) t\right)^{2} \\
-2{ }_{-}{ }^{2} a_{4}^{2}\left(x+\left(8064 a_{4}-72 \alpha a_{6}-\frac{3}{2} g_{4}\right.\right. & \left.\left.\left.+2520 a_{4}^{2}-60 a_{4} \alpha\right) t\right)+a_{4-}^{2} \mathrm{Cl}^{2}\right)^{-2} .
\end{aligned}
$$

The graph of (31) is illustrated for selected values of coefficients given in Figure 1.

Example 2. We, next, consider the auiliary equation with sixdegree nonlinearity, that is,

$$
\left(\frac{d z}{d \xi}\right)^{2}=a_{2} z^{2}(\xi)+a_{6} z^{6}(\xi)
$$




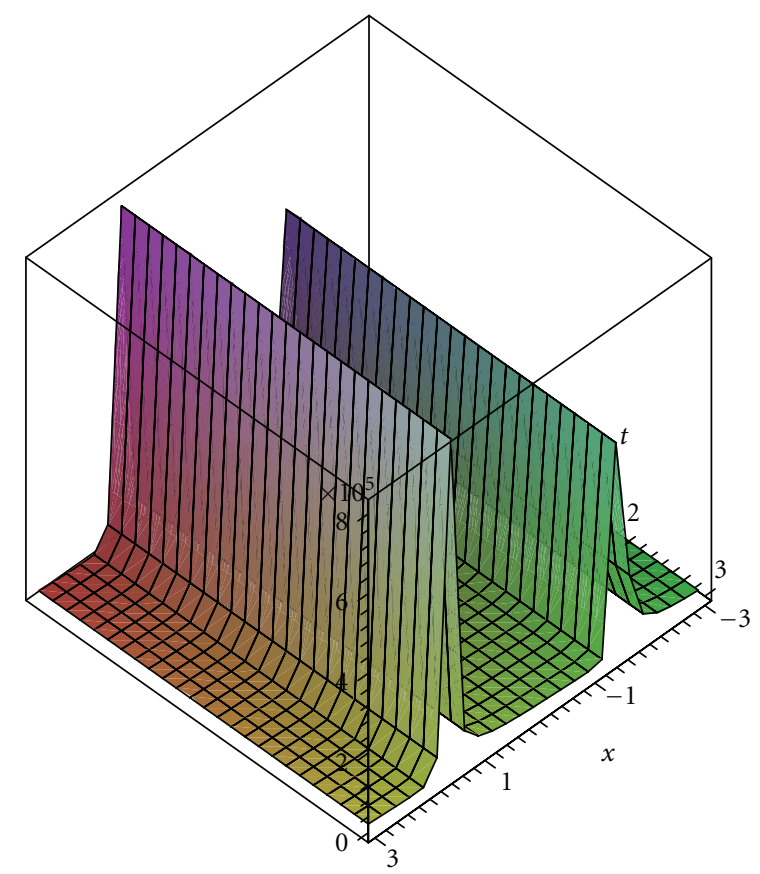

Figure 1: Graph of (31) for $g_{4}=1, \alpha=\mu=1, a_{4}=\tan x, a_{6}=-1$.

in Cases 1-8. The solution of (32)

$z(\xi)$

$$
\begin{aligned}
= & e^{\left(\sqrt{a_{2}}\left(-\xi+c_{1}\right)\right)} 2^{1 / 4} \\
& \times\left(a_{6} e^{\left(4 \sqrt{a_{2}}\left(-\xi+c_{1}\right)\right)}\left(a_{2} \operatorname{Lambert} W\left(-\frac{a_{6} e^{\left(4 \sqrt{a_{2}}\left(-\xi+c_{1}\right)\right)}}{2 a_{2}}\right)\right)^{-1}\right)^{-1 / 4}
\end{aligned}
$$

is, we belive, a new solution in the literature.

Substituting (26) and (32) into (25) and letting each coefficient of $z^{i}(\xi) \sqrt{a_{2} z^{2}(\xi)+a_{6} z^{6}(\xi)},(0 \leq i \leq 8)$ to be zero, we obtain

$$
\begin{gathered}
50 \alpha-3188 a_{2}=0, \\
30 \alpha a_{2}-354 a_{2}^{2}-4 c=0 .
\end{gathered}
$$

Solving the system (34) by the aid of Maple 13, we can determine the coefficients:

$$
\begin{gathered}
c=\frac{487125 \alpha^{2}}{5081672}, \quad g_{0}=g_{0}, \quad g_{1}=g_{1}, \quad g_{2}=g_{2}, \\
g_{3}=g_{3}, \quad g_{4}=g_{4}, \quad \mu=\mu, \\
a_{2}=\frac{25 \alpha}{1594}, \quad a_{6}=a_{6} .
\end{gathered}
$$

Substituting the above coefficients into ansatz (26) with the solution (33) of auxiliary equation, we obtain another new solution of Kawahara equation:

$u(x, t)$

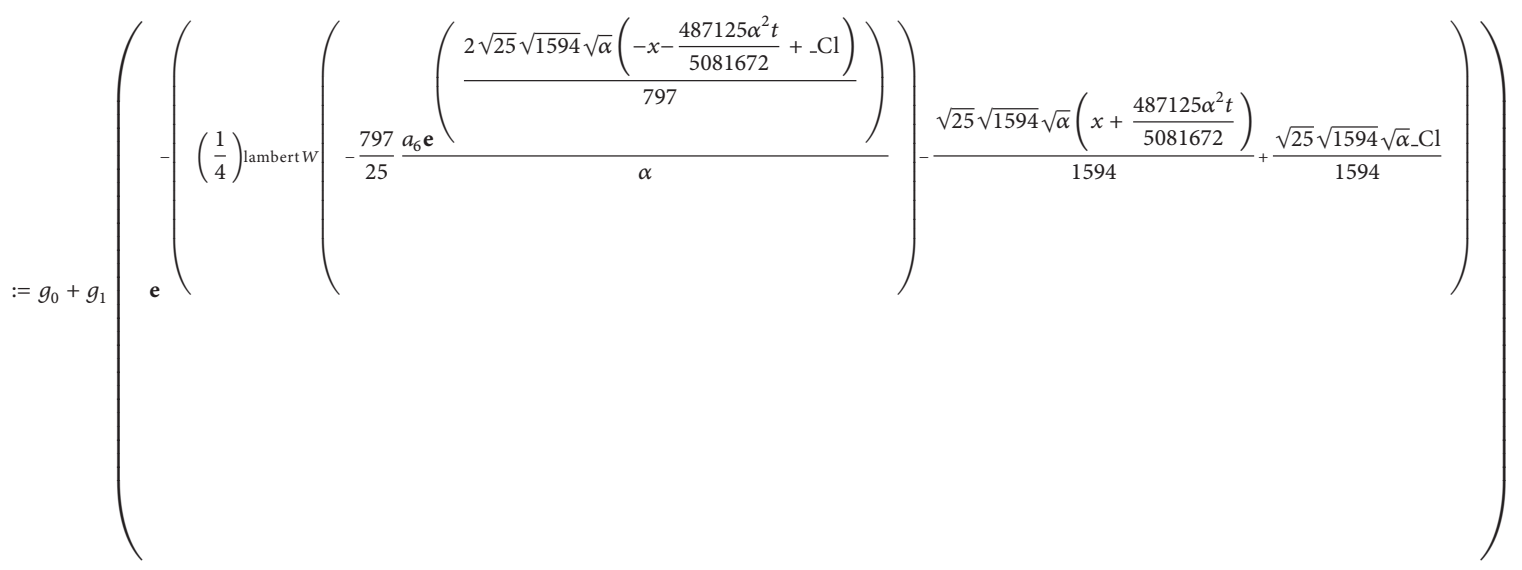



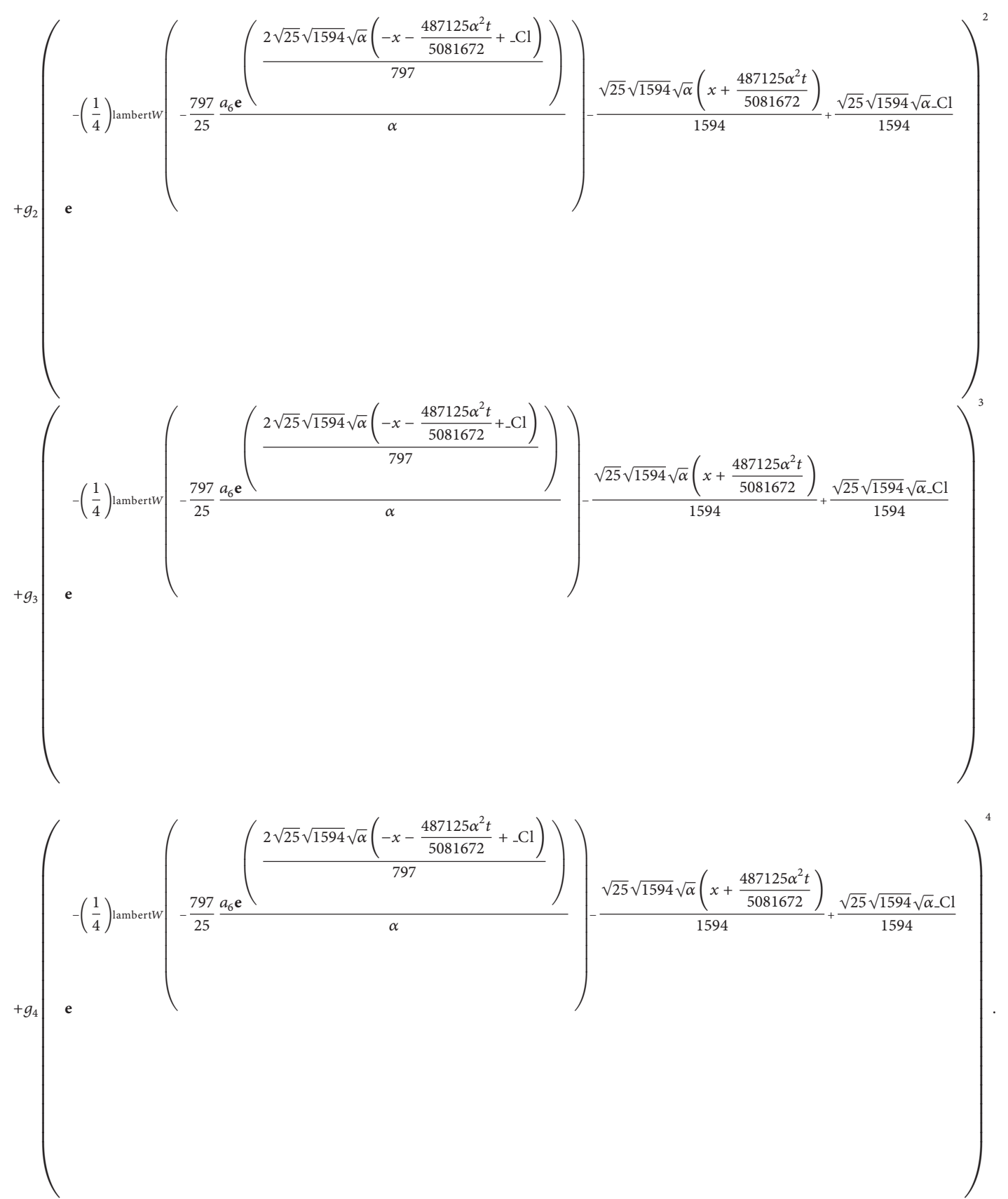

The graph of (36) is illustrated for selected values of coefficients given in Figure 2.

\section{Conclusion}

As is seen, the key idea of obtaining new travelling wave solutions for the nonlinear equations is constructing different types of solutions of the given auxiliary equation. In this paper, the exact solutions of the auxiliary equation with sixdegree nonlinearity (6) is

$$
\left(\frac{d z}{d \xi}\right)^{2}=a_{0}+a_{2} z^{2}(\xi)+a_{4} z^{4}(\xi)+a_{6} z^{6}(\xi),
$$

where $a_{i}(i=0,2,4,6)$ are real constants, used to construct the solutions of Klein-Gordon equation [32]. Using these solutions, we have successfully obtained some new exact periodic solutions of the Kawahara equation. For comparative purposes, it is noticeable that $\mathrm{Wu}$ et al. in [20] and $\mathrm{Lv}$ et al. in [23] have utilized (5) for $a_{i}(i=$ $0,2,4)$, and Sirendaoreji in [21] and Jang in [22] have applied the ansatz $(d z / d \xi)^{2}=a_{2} z^{2}(\xi)+a_{3} z^{3}(\xi)+a_{4} z^{4}(\xi)$ which only covers, at most, fourth-order nonlinear term in auxiliary ordinary differential equation, and therefore their solutions are limited in the quantity to the order considered. 


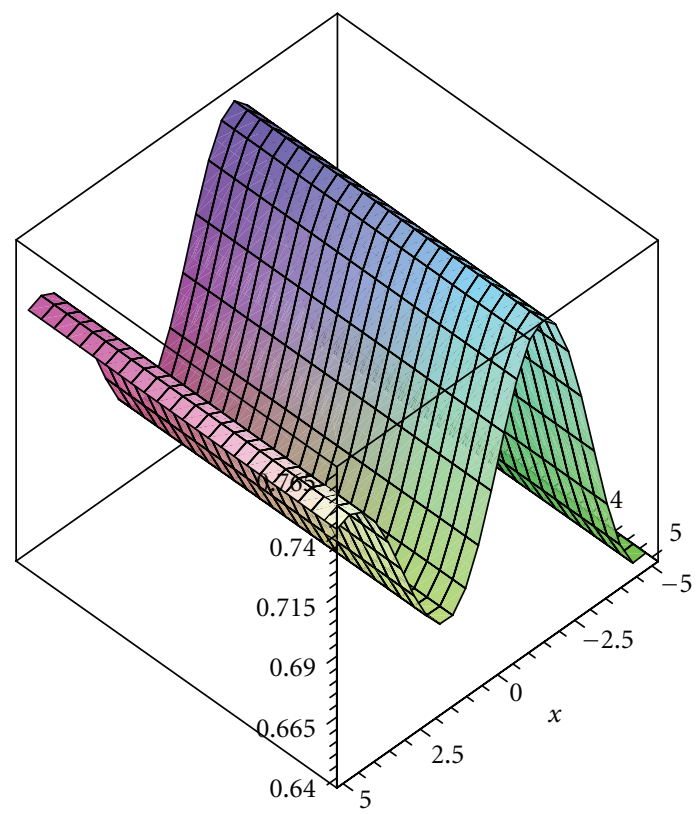

FigURE 2: Graph of (36) for $\alpha=1 / 2, a_{6}=g_{0}=g_{2}=1, g_{1}=g_{3}=-1$, $g_{4}=\sin x$.

In this letter, we have obtained exact solutions of the auxiliary equation with sixth-degree nonlinearity (6) for distinct cases (see, Cases 1-8). Therefore, our approach gives additional new solutions beside the solutions obtained via fourth order auxiliary equation, and theoretically, some of our solutions may coincide with the solutions of Wu et al. in [20], Sirendaoreji in [21], Jang in [22], Lv et al. in [23], and some cases of Yomba [24] for certain choice of the parameters which we have left as an exercise.

However, it is well known that different types of auxiliary equations produce new travelling wave solutions for many nonlinear problems. Hence this also is our future work. The presented method could lead to finding new exact travelling wave solutions for other nonlinear problems. (33) of auxiliary equation, we obtain another new solution of Kawahara

\section{References}

[1] G. B. Whitham, "A general approach to linear and nonlinear waves using a Lagrangian," Journal of Fluid Mechanics, vol. 22, pp. 273-283, 1965.

[2] R. Knobel, An Introduction to the Mathematical Theory of Waves, American Mathematical Society, Providence, RI, USA, 2000.

[3] S. S. Shen, A Course on Nonlinear Waves, Kluwer, Dordrecht, The Netherlands, 1994.

[4] C. Yong, L. Biao, and Z. Hong-Qing, "Generalized Riccati equation expansion method and its application to the Bogoyavlenskii's generalized breaking soliton equation," Chinese Physics, vol. 12, no. 9, pp. 940-946, 2003.

[5] Y. Zhou, M. Wang, and Y. Wang, "Periodic wave solutions to a coupled KdV equations with variable coefficients," Physics Letters A, vol. 308, no. 1, pp. 31-36, 2003.

[6] G. Cai, Q. Wang, and J. Huang, "A modified F-expansion method for solving breaking soliton equation," International Journal of Nonlinear Science, vol. 2, pp. 122-128, 2006.
[7] X. Zeng and X. Yong, "A new mapping method and its applications to nonlinear partial differential equations," Physics Letters A, vol. 372, no. 44, pp. 6602-6607, 2008.

[8] X. Yong, X. Zeng, Z. Zhang, and Y. Chen, "Symbolic computation of Jacobi elliptic function solutions to nonlinear differential-difference equations," Computers \& Mathematics With Applications, vol. 57, no. 7, pp. 1107-1114, 2009.

[9] T. Öziş and I. Aslan, "Exact and explicit solutions to the (3 +1 )-dimensional Jimbo-Miwa equation via the Exp-function method," Physics Letters A, vol. 372, no. 47, pp. 7011-7015, 2008.

[10] T. Öziş and C. Köroğlu, "A novel approach for solving the Fisher equation using Exp-function method," Physics Letters A, vol. 372, no. 21, pp. 3836-3840, 2008.

[11] A. Yildirım and Z. Pinar, "Application of the Exp-function method for solving nonlinear reactiondiffusion equations arising in mathematical biology," Computers and Mathematics with Applications, vol. 60, no. 7, pp. 1873-1880, 2010.

[12] W. X. Ma, T. Huang, and Y. Zhang, "A multiple Exp-function method for nonlinear differential equations and its applications," Physica Scripta, vol. 82, Article ID 065003, 8 pages, 2010.

[13] T. Öziş and I. Aslan, "Application of the $G^{\prime} /$ G-expansion method to Kawahara type equations using symbolic computation," Applied Mathematics and Computation, vol. 216, no. 8, pp. 2360-2365, 2010.

[14] T. Öziş and I. Aslan, "Symbolic computation and construction of new exact traveling wave solutions to Fitzhugh-Nagumo and Klein-Gordon equations," Zeitschrift für Naturforschung, vol. 64, no. 1-2, pp. 15-20, 2009.

[15] I. Aslan and T. Öziş, "Analytic study on two nonlinear evolution equations by using the $\left(G^{\prime} / G\right)$-expansion method," Applied Mathematics and Computation, vol. 209, no. 2, pp. 425-429, 2009.

[16] T. Öziş and M. Aslan, "Symbolic computations and exact and explicit solutions of some nonlinear evolution equations in mathematical physics," Communications in Theoretical Physics, vol. 51, no. 4, pp. 577-580, 2009.

[17] H. Zhang, "A note on some sub-equation methods and new types of exact travelling wave solutions for two nonlinear partial differential equations," Acta Applicandae Mathematicae, vol. 106, no. 2, pp. 241-249, 2009.

[18] B. Li, Y. Chen, and Y. Q. Li, "A generalized sub-equation expansion method and some analytical solutions to the inhomogeneous higher-order nonlinear Schrödinger equation," Zeitschrift für Naturforschung, vol. 63, no. 12, pp. 763-777, 2008.

[19] E. Yomba, "The modified extended Fan sub-equation method and its application to the (2+1)-dimensional Broer-KaupKupershmidt equation," Chaos, Solitons and Fractals, vol. 27, no. 1, pp. 187-196, 2006.

[20] G. J. Wu, J. H. Han, W. L. Zhang, M. Zhang, and J. M. Wang, "New periodic wave solutions to generalized Klein-Gordon and Benjamin equations," Communications in Theoretical Physics, vol. 48 , no. 5, pp. 815-818, 2007.

[21] Sirendaoreji, "Auxiliary equation method and new solutions of Klein-Gordon equations," Chaos, Solitons and Fractals, vol. 31, no. 4, pp. 943-950, 2007.

[22] B. Jang, "New exact travelling wave solutions of nonlinear Klein-Gordon equations," Chaos, Solitons and Fractals, vol. 41, no. 2, pp. 646-654, 2009.

[23] X. Lv, S. Lai, and Y. Wu, "An auxiliary equation technique and exact solutions for a nonlinear Klein-Gordon equation," Chaos, Solitons and Fractals, vol. 41, no. 1, pp. 82-90, 2009. 
[24] E. Yomba, "A generalized auxiliary equation method and its application to nonlinear Klein-Gordon and generalized nonlinear Camassa-Holm equations," Physics Letters A, vol. 372, no. 7, pp. 1048-1060, 2008.

[25] J. Nickel, "Elliptic solutions to a generalized BBM equation," Physics Letters A, vol. 364, no. 3-4, pp. 221-226, 2007.

[26] E. T. Whittaker and G. N. Watson, A Course of Modern Analysis, Cambridge University Press, Cambridge, UK, 1927.

[27] E. Fan, "Multiple travelling wave solutions of nonlinear evolution equations using a unified algebraic method," Journal of Physics A, vol. 35, no. 32, pp. 6853-6872, 2002.

[28] E. Yomba, “The extended Fan's sub-equation method and its application to KdV-MKdV, BKK and variant Boussinesq equations," Physics Letters A, vol. 336, no. 6, pp. 463-476, 2005.

[29] Z. Yan, "An improved algebra method and its applications in nonlinear wave equations," Chaos, Solitons and Fractals, vol. 21, no. 4, pp. 1013-1021, 2004.

[30] M. A. Abdou, "A generalized auxiliary equation method and its applications," Nonlinear Dynamics, vol. 52, no. 1-2, pp. 95-102, 2008.

[31] D. J. Huang and H. Q. Zhang, "New exact travelling waves solutions to the combined KDV-MKDV and generalized Zakharov equations," Reports on Mathematical Physics, vol. 57, no. 2, pp. 257-269, 2006.

[32] Z. Pınar and T. Öziş, "An observation on the periodic solutions to nonlinear physical models by means of the auxiliary equation with a sixth-degree nonlinear term," Communications in Nonlinear Science and Numerical Simulation, 2013. 


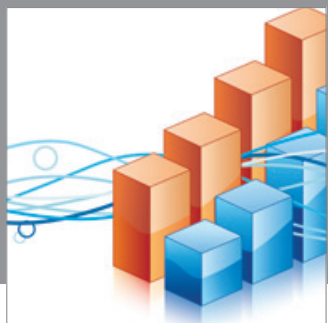

Advances in

Operations Research

mansans

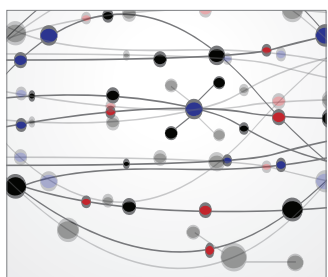

The Scientific World Journal
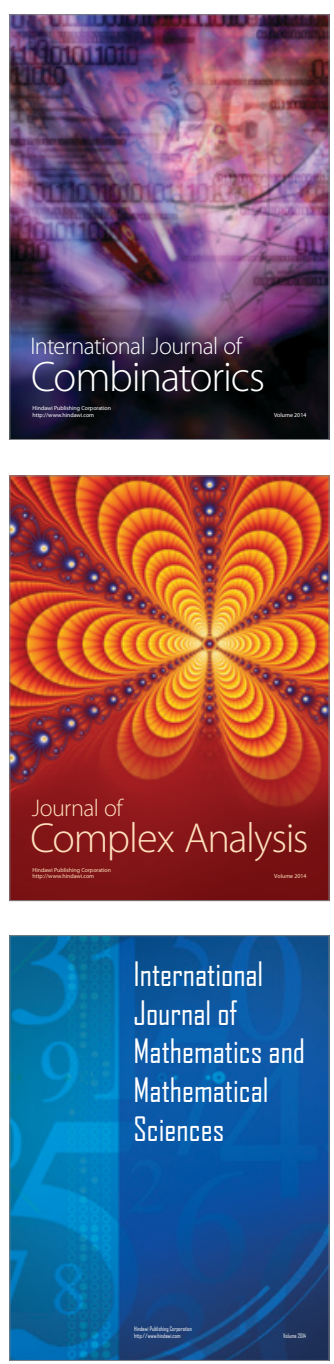
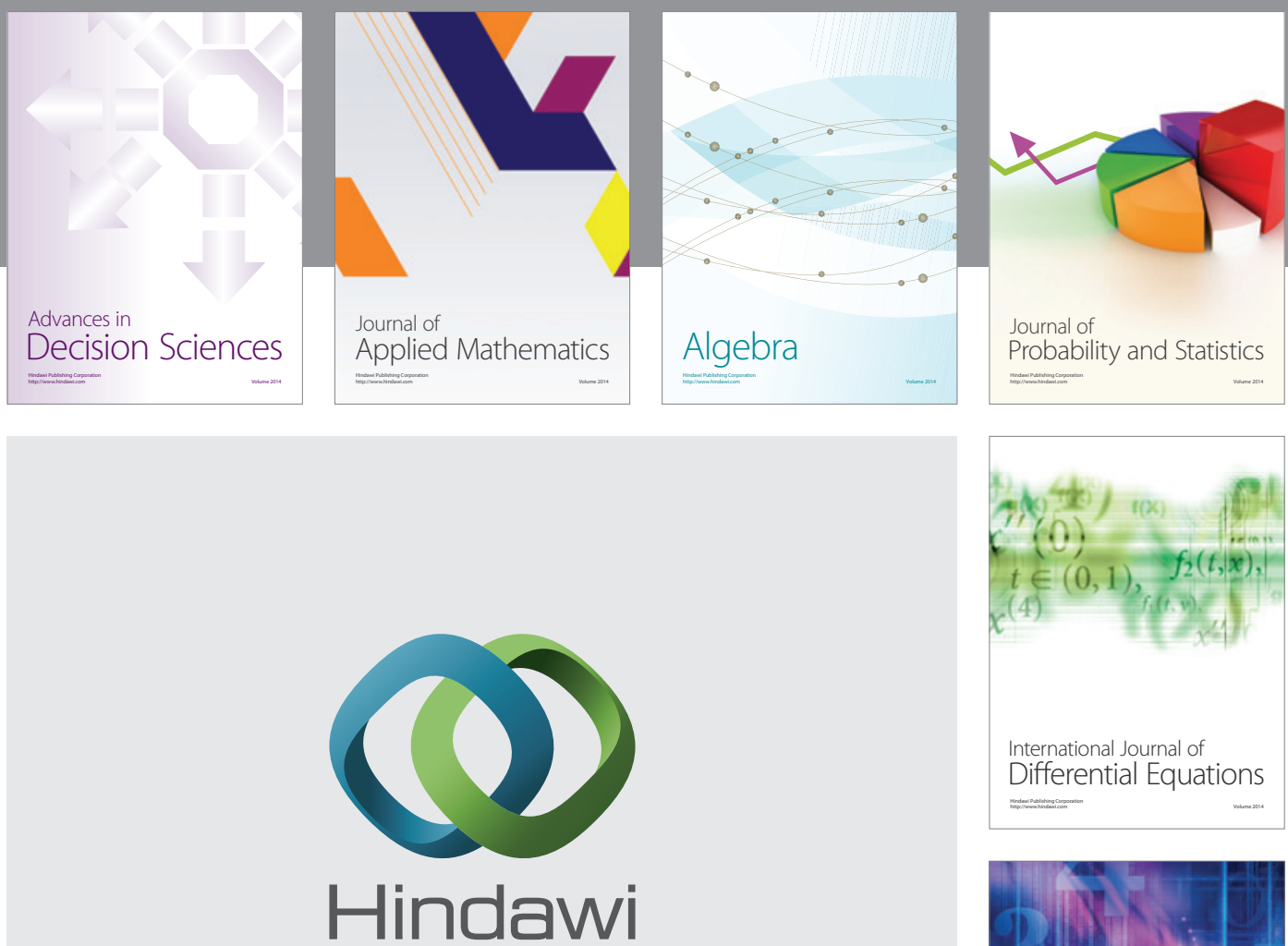

Submit your manuscripts at http://www.hindawi.com
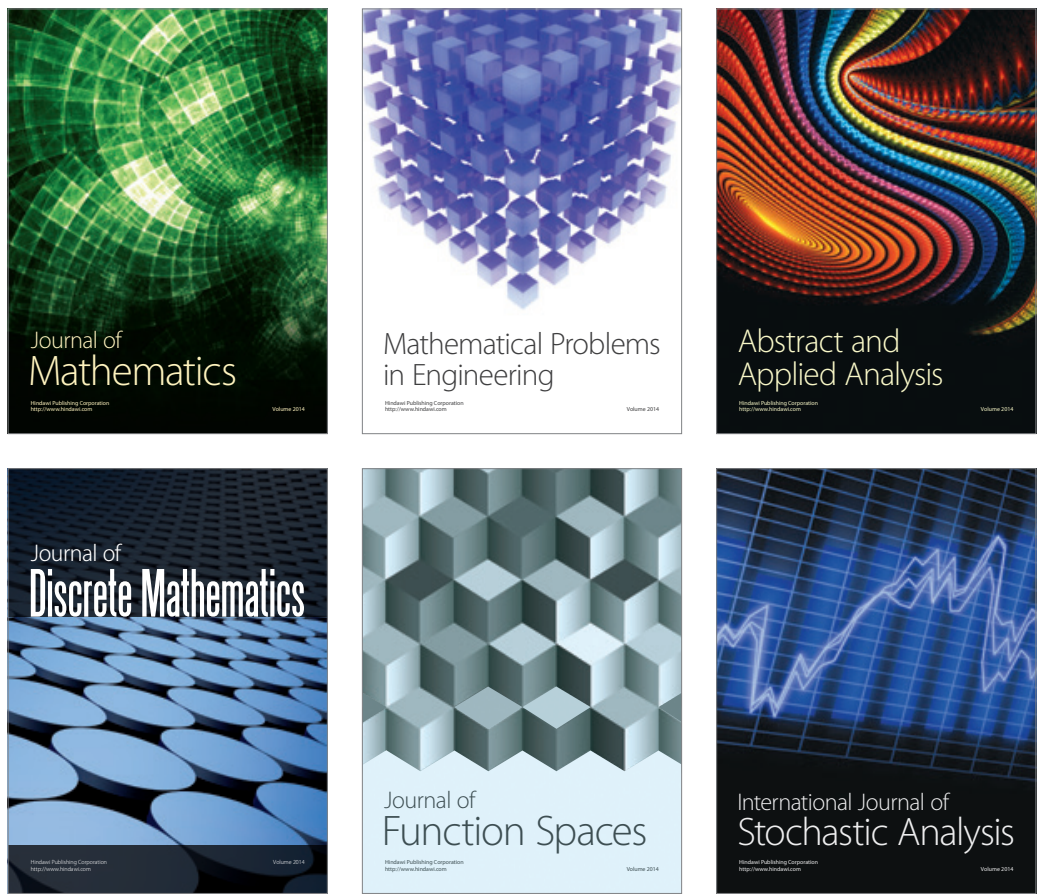

Journal of

Function Spaces

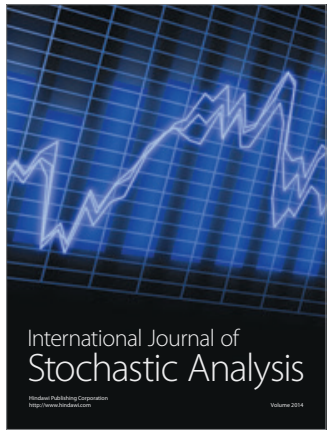

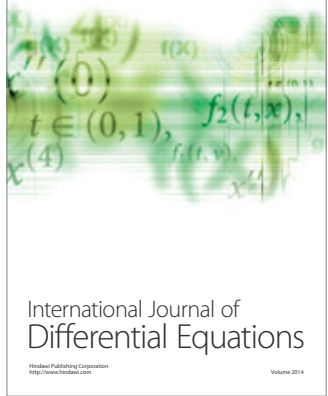
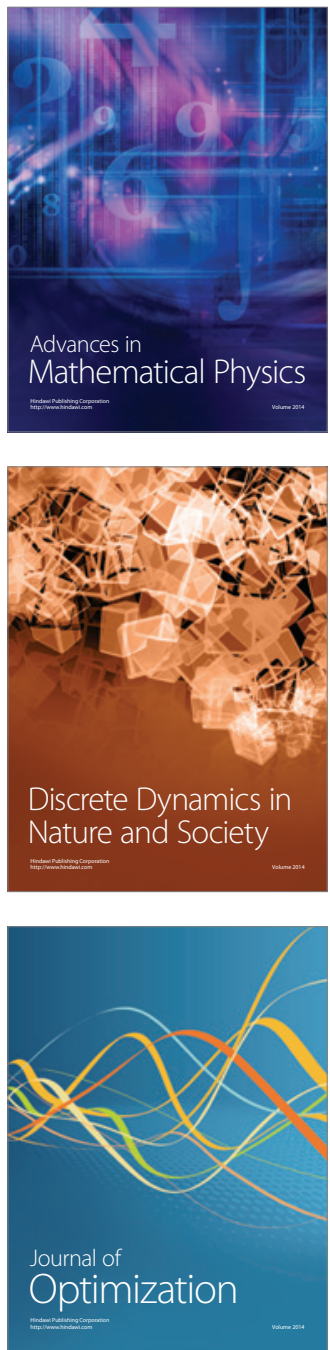